\title{
Interpretation of the Be star HD 163868 oscillation spectrum based on the MOST observations
}

\author{
W. A. Dziembowski, ${ }^{1,2}$ J. Daszyńska-Daszkiewicz, ${ }^{1,3}$ A. A. Pamyatnykh ${ }^{1,4}$ \\ ${ }^{1}$ Copernicus Astronomical Center, Bartycka 18, 00-716 Warsaw, Poland \\ ${ }^{2}$ Warsaw University Observatory, Al. Ujazdowskie 4, 00-478 Warsaw, Poland \\ ${ }^{3}$ Astronomical Institute, Kopernika 11, 51-622 Wroctaw, Poland \\ ${ }^{4}$ Institute of Astronomy, Pyatnitskaya Str. 48, 109017 Moscow, Russia
}

On the basis of MOST photometry, Walker et al. (2005) detected a large number of significant peaks in the oscillation spectrum of HD 163868 and provided an interpretation for some of them in terms of high-order $g$ modes. However, after conducting a stability survey for low degree modes in a model that seems appropriate for the star they were unable to explain the low frequency part of the spectrum, where the amplitudes are the highest.

In the observed frequency spectrum, shown in the upper panel of Fig. 1, we see three groups of modes. According to Walker et al. (2005), the highest frequency group may be explained by excitation of prograde $\ell=2, m=2 \mathrm{~g}$ modes and the group at intermediate frequencies by prograde $\ell=1 \mathrm{~g}$ modes. Because individual modes are no longer described by single spherical harmonics, the $\ell$ values are used only for mode identification. These authors found a large number of unstable modes in these two frequency ranges. They also found an instability of retrograde $m=-1 \mathrm{r}$ modes in the frequency range which extends down to $0.43 \mathrm{c} / \mathrm{d}$, thus encompassing part of the lowest frequency group. In their study they rely on the truncated Legendre expansion.

We made our stability survey for nearly identical model but relying on the traditional approximation. Our results, presented in the lower panel of Fig. 1, differ significantly from those of Walker et al. (2005). We do not find any unstable prograde $\ell=1 \mathrm{~g}$ mode, instead we find many other unstable modes in the whole frequency range. Taking into account visibility conditions, which depend on mode geometry and the aspect angle, $i$, we found that occurrence of peaks in the three separate frequency ranges may be understood if $i \approx 90^{\circ}$. Then our interpretation of the highest frequency group is $\ell=2, m=2$ (the same as proposed by Walker et al.), of the intermediate frequency group is $\ell=2, m=0$ and $m=-2$, and of the lowest frequency group is $\ell=1, m=-1$. The best fit is obtained assuming a rotation velocity of about $270 \mathrm{~km} / \mathrm{s}$, that is somewhat lower than used in Fig. 1 . If $i \approx 55^{\circ}$, as Walker et al. (2005) adopted, then there would be no explanation for the gap around the frequency of $1 \mathrm{c} / \mathrm{d}$, where we would expect to see the $\ell=1, m=0$ modes, which are unstable and have good visibility at this aspect. A detailed description of our calculations has been published elsewhere (Dziembowski et al. 2007).

Acknowledgments. The authors acknowledge partial financial support from the HELAS project and from the Polish MNil grant No. 1 P03D 02128.

\section{References}

Dziembowski W. A., Daszyńska-Daszkiewicz J., Pamyatnykh A. A., 2007, MNRAS, 374, 248

Walker G. A. H., Kuschnig R., Matthews J. M., et al., 2005, ApJ, 635, L77 


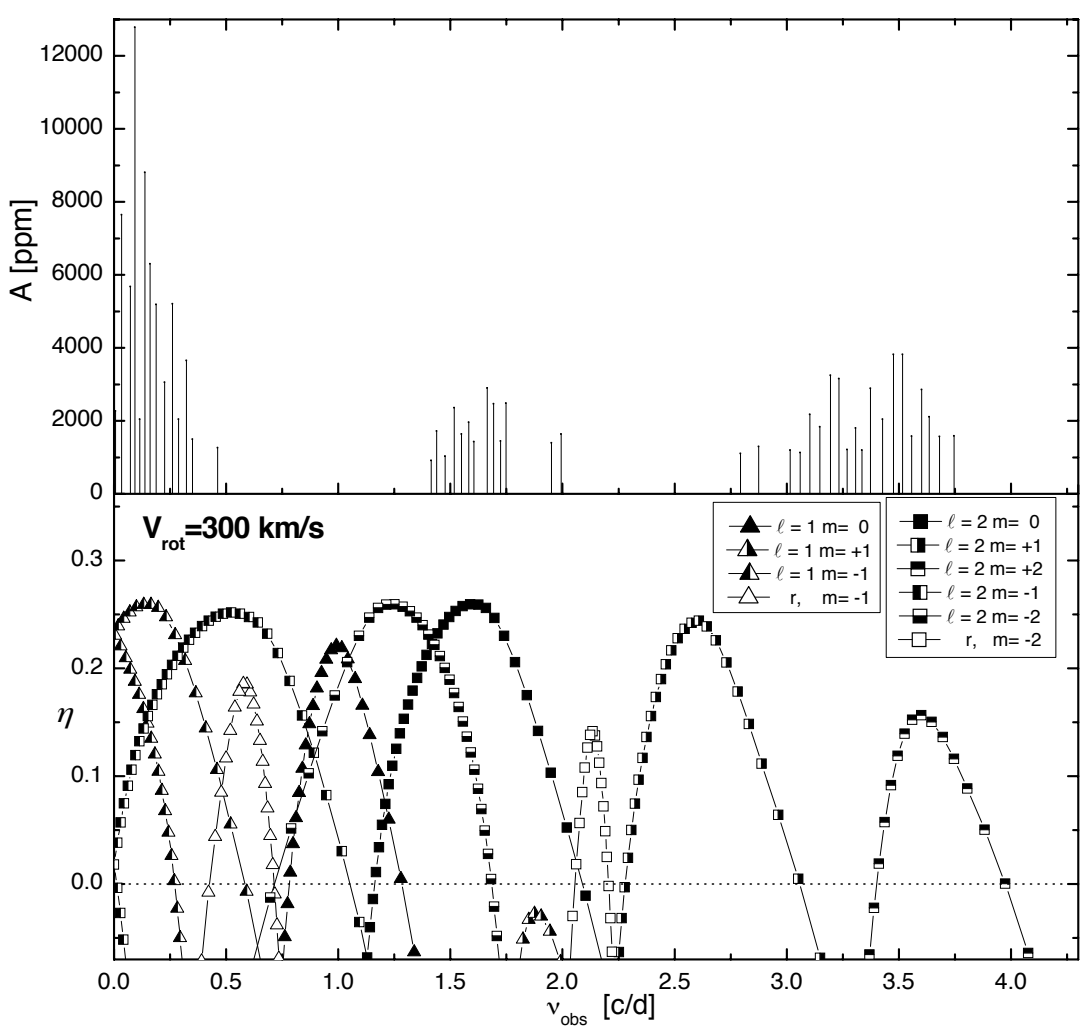

Figure 1: Top: Frequency spectrum of HD 163868. Bottom: Normalized growth rates, $\eta(\eta>0$ means instability), for low-degree modes in the model of HD 163868. 